\title{
Research on RFID collision detection algorithm based on the underdetermined blind separation
}

\author{
XiaoHui $\mathrm{CHENG}^{1}$,Chuan LIU $^{2}$ \\ ${ }^{1,2}$ College of Information Science and Engineering ,Guilin University Of Technology,GuiLin Gu \\ angXi 541006,China \\ acxiaohui@glut.edu.cn, ${ }^{\mathrm{b}} 1044785042 @ q q . c o m$
}

\begin{abstract}
Key Words:RFID;blind source separation;non fully sparse
\end{abstract}
\begin{abstract}
Aiming at the collision problem of RFID tag communication, we proposed a new method for identifying the RFID recognition of the blind separation of source signals, which is based on the label collision and recovery. In the case that the number of tags is more than that of the reader,we established a RFID anti collision model based on the blind separation.In the same time considering the non full sparsity of RFID signal,we used an improved two step extraction method.Firstly We estimated the base vector of part of the source signal by the sample of the unit interval of signal.Then we extracted the corresponding source signal with the minimal interference so that the necessary conditions for the recognition of the mixed matrix can be avoided.After that we have carried out a simulation comparison in terms of throughput.The result showed that the method of this paper is better than the conventional blind separation algorithm and ALOHA algorithm in terms of throughput and it is more suitable for the collision identification and recovery of RFID non fully sparse signal.
\end{abstract}

\section{Introduction}

Radio frequency identification, RFID (Frequency Identification Radio) technology, which is also known as radio frequency identification.But at present, it still has some bottleneck problems which restrict its development, such as the security problem of information transmission, the protocol and the collision problem and so on.Collision problem, such as in the the RFID system, when the same frequency of the label in the same reader identification range, there will be a problem for the identification of tags. This problem greatly reduces the work efficiency of the RFID system.An effective collision avoidance strategy can ensure that each label is read correctly, improve resource utilization, so that the reader and multiple tags can communicate effectively.

The anti collision algorithm which are mainly applied in the current RFID system are the following ones: the space division multiple access, time division multiple access, frequency division multiple access, code division multiple access.Time slot Aloha algorithm based on time division multiple access and binary search algorithm, is widely used in RFID tag anti collision algorithm.In the paper ${ }^{[1]}$, a new Aloha algorithm based on time slot is proposed, but it can only solve the problem of incomplete collision.In the paper ${ }^{[2]}$ a binary tree algorithm is proposed, which can reduce the search time and transmission delay effectively, but it still has the problem of low utilization rate of channel, long recognition time .In the paper ${ }^{[3]}$, a code division multiple access method is used to avoid collision problem, but when the number of tags is very large, due to the allocation of waiting, the resource reading efficiency is still relatively low.

Blind Source Separation (BSS:) is the process of separating the source signals from the observed signals without knowing the source signal and the transmission channel parameters.By using this 
technique, the original signal can be effectively separated from the mixed impact signal, so as to prevent the impact of the collision.By this technique, the identification time is short and when the number of tags is relatively large, the efficiency is still relatively high.Blind source separation algorithm which is mainly based on sparsity uses two step method ,such as DUET, TIFROM, single source, ${ }^{[4-5]}$, MRISSI, and so on.However, RFID is usually used in ASK or PSK modulation, which is not fully sparse.The sparse algorithms above are often unable to complete the identification of aliasing matrix, the source of the separation can not be used in sparse decomposition method, so it can not be applied to the RFID signal blind source separation.In view of this situation, this paper proposes a RFID anti collision method based on the non fully sparse property of the blind.In the premise that the number of the readers is less than the number of tags, we set up a multi label communication model, and use the unit interval of signal, a kind of non sparse algorithm to deal with the problem of label collision.And in the same time we used matlab to detect the performance of the algorithm.

\section{RFID anti collision model}

In this paper, we consider the case of multiple tags, which requires the establishment of a multi input output (MIMO) RFID model.Assuming that the reader has a $\mathrm{M}$ antenna, there is a $\mathrm{N}$ tag which communicate with the reader. A collision occurs when the tag signal is synchronized to the reader.

Set the reflected signal : $s(t)=\left[s_{1}(t), s_{2}(t), \ldots, s_{N}(t)\right]^{T}, s_{j}(t)$ represent the signal which was launched from the $\mathrm{j}$ tag.

The signal received by the reader antenna is expressed as:

$x(t)=\left[x_{1}(t), x_{2}(t), x_{3}(t), \ldots, x_{M}(t)\right]^{T}, x_{i}(t)$ is The signal received by the reader on the no.i antenna.Under the condition of flat fading,as each pair of antennas can be equivalent to a Rayleigh fading channel, the sub channels in the model can be expressed as:

$r_{i, j}(\tau, t)=r_{i, j} \delta\left(\tau-\tau_{0}\right)$;

$\mathrm{i}=1,2 \ldots \mathrm{M}, \mathrm{j}=1,2 \ldots \mathrm{N},\left|r_{i, j}\right|$ Obeyed Rayleigh distribution ,then by the channel matrix of the model : $R=\left(r_{i, j}\right)_{M \times N}$, we can get :

$$
x(t)=R s(t)+Z
$$

$\mathrm{Z}$ is Zero mean Gauss white noise matrix.

The basic mathematical model of blind source separation is:

$$
\mathrm{x}(\mathrm{t})=\operatorname{As}(\mathrm{t})+\mathrm{v}(\mathrm{t})
$$

Among it, $\quad x(t)=\left[x_{1}(t), x_{2}(t), \ldots, x_{M}(t)\right]^{T} \in C^{M} \quad$ is $\quad$ observation signal vector, $s(t)=\left[s_{1}(t), s_{2}(t), \ldots, s_{N}(t)\right]^{T} \in C^{N}$ is mutually independent source signals;A is $M \times N$ Real number matrix which is called mixed matrix.The element is $a_{i, j} ; \mathrm{v}(\mathrm{t})$ is Gauss white noise.Theoretical analysis of this paper will not consider Gauss white noise.

Combined (1) (2), when the RFID R model in the weak channel MIMO as the blind source 
separation of the mixed matrix A, (1) and (2) are same.So blind source separation method can be used to deal with the problem of multi-antenna and multi-tag RFID collision.In the RFID MIMO system we have constructed in this paper, when the number of tags is less than the number of readers $(\mathrm{M}<\mathrm{N})$, that is the case of the blind source separation in the blind source separation, so we use the blind separation of the case to solve the collision problem in this paper.

\section{RFID collision detection algorithm based on the underdetermined blind separation}

In recent years, the algorithm of the blind source separation is mainly based on the sparsity of the signal, using the "two step method" to get the source signal.Because of the non complete sparse characteristics of RFID, this paper uses a new two step solution for RFID signal with no correlation between the source signals.First, a single source region sample is used to estimate the base vector, and the corresponding source is extracted, which is independent of the other basic vector.In this way, the mixed matrix can not be fully estimated, and the target of the extraction is achieved.

\subsection{Estimation of base vector}

The mixed matrix A can be expanded to: $A=\left[a^{1}, a^{2}, \ldots, a^{n}\right]$,vector $a^{j}$ is referred to the base vector of the no.j source.Set $\left\|a^{j}\right\|=1$ ( $\left\|a^{j}\right\|$ is the vector length), Under the premise of no consideration of the noise, the formula (2) can be rewritten as

$$
x(t)=\sum_{j=1}^{n} a^{j} S_{j}(t)
$$

Three conditions are assumed: (1) The arbitrary two columns of the mixing matrix are linearly independent; (2) The source signals are uncorrelated with each other and have zero mean;(3)Rank of $\mathrm{A}$ in the mixed matrix meets $\operatorname{rank}(\mathrm{A})=\mathrm{m}$.

According to the paper ${ }^{[6]}$, the estimation of the mixed matrix requires that all sources have some single source region, and some basic vector will not be estimated in the condition of non total sparsity.According to the definition of base vector:

Definition 1:After the mixing matrix has been spared ,for a given sample interval $\left[t_{1}, t_{2}\right]$,If and

only a source is nonzero, the other sources are 0 , called interval $\left[t_{1}, t_{2}\right]$ is the mixture of overlapping signals of single source intervals (single source interval, SSI).

Obviously, if $\left[t_{1}, t_{2}\right]$ is a single source range, $x(t)=a^{j} s_{j}(t)$.The directions of vector $x(t)$ and $a^{j}$ are the same or the opposite.They are on the same line.Set

$u(t)=x(t) /\|x(t)\|, u(t)=\left[u_{1}(t), \ldots u_{m}(t)\right]^{T}$;assume

$d^{t}=u(t) \cdot \operatorname{sign}\left(u_{k}(t)\right)-u(t+1) \operatorname{sign}\left(u_{k+1}(t)\right)\left(k=\arg \max \left|u_{i}(t)\right|\right), \operatorname{sign}(\cdot)$ represents a symbolic

function.In this case we can get the nature:If the interval $\left[t_{1}, t_{2}\right]$ is a single source interval,in $t \in[t 1, t 2)$, vector $d^{t}$ is 0 . 
According to its properties, the SAMTD algorithm can be used to estimate the total or partial basis vectors, which is the first step of the two step extraction method.

However, in the assumption that the non aliasing matrix can be recovered, it will not be able to judge whether the application of sparse decomposition method.Next a two step blind extraction algorithm is proposed, which extracts the j source signal directly and does not depend on the other base vectors, and the assumption that the aliasing matrix can be avoided.

\subsection{Source signal extraction}

After estimating the base vector $a^{j}$, we need to discuss how to recover the no.J source.The signal is divided into several regions, then we analyze the sample in any interval $\left[t_{1}, t_{2}\right]$.

On the premise of no noise, on the interval $\left[t_{1}, t_{2}\right]$, set up a vector $w_{j}=\left\lfloor w_{j 1}, \ldots, w_{j m}\right\rfloor$ to make:

$$
z_{j}(t)=w_{j} x(t)
$$

The general goal is $z_{j}(t)$, which is closest to the source $s_{j}(t)$, in theory can sometimes be equal to the source $s_{j}(t)$.

The vector $a^{j}$ has been known to be nonzero, and the no.K component can be set: $a_{k j} \neq 0$.We construct matrix $B=\left[b_{1}, \ldots, b_{m-1}\right]^{T}$ :

$$
B=\left[\begin{array}{ccccccc}
-a_{k j} & 0 & \cdots & a_{1 j} & 0 & \cdots & 0 \\
\vdots & \ddots & \vdots & \vdots & \vdots & \vdots & \vdots \\
0 & \cdots & -a_{k j} & a_{k-1, j} & 0 & \vdots & 0 \\
0 & \cdots & 0 & a_{k+1, j} & -a_{k j} & \cdots & 0 \\
\vdots & \vdots & \vdots & \vdots & \vdots & \ddots & 0 \\
0 & \cdots & 0 & a_{m j} & 0 & \cdots & -a_{k j}
\end{array}\right]_{(m-1) \times m}
$$

We can know that $\operatorname{rank}(\mathrm{B})=\mathrm{m}-1$,each line of $b_{i}(\mathrm{i}=1,2, \ldots, \mathrm{m}-1)$ is orthogonal to the vector $a^{j}$.Calculating $y(t)=B x(t), y(t)=\left[y_{1}(t), \ldots, y_{m-1}(t)\right]^{T}$.Take type(3) into the type above,we can get $y_{i}(t)$ : $\quad y_{i}(t)=\sum_{\substack{k=1 \\ k \neq j}}^{n} b_{i} a^{k} s_{k}(t)$

We can know the each component of $\mathrm{y}(\mathrm{t})$ does not contain the no.J source.

We projected vector $\mathrm{x}(\mathrm{t})$ onto the base vector $a^{j}$.We can get $x_{0}(t)=\left(a^{j}\right)^{T} x(t)$. Assuming $\lambda=\left[\lambda_{1}, \lambda_{2}, \cdots, \lambda_{m-1}\right]$,reduce or eliminate the interference from other sources from $x_{0}(t), \hat{s}_{j}(t)=x_{0}(t)-\lambda y(t), \hat{s}_{j}(t)$ is the estimated signal. 
Discuss the average power $E\left\lfloor\left|\hat{s}_{j}(t)\right|^{2}\right\rfloor$ of signal $\hat{s}_{j}(t)$ in the interval $\left[t_{1}, t_{2}\right]$.Then $E\left\lfloor\left|\hat{s}_{j}(t)\right|^{2}\right\rfloor=E\left\lfloor x_{0}(t)-\left.\lambda y(t)\right|^{2}\right\rfloor$.

According to the irrelevance between the source signal, $E\left[\left|s_{j}(t) s_{i}(t)\right|^{2}\right]=0(i \neq j)$, get:

$$
E\left[\left|\hat{s}_{j}(t)\right|^{2}\right]=E\left[\left|s_{j}(t)\right|^{2}\right]+\sum_{\substack{i=1 \\ i \neq j}}^{m}\left[\left(a^{j}\right)^{T} a^{i}-\sum_{k=1}^{m-1} \lambda_{k} b_{k} a^{i}\right]^{2} E\left[\left|s_{i}(t)\right|^{2}\right]
$$

In (6), the source which we want to item to be extracted is the first item, and the interference source is second, we want to make the second minimum.Obviously the first item has nothing to do with the parameter, so $\lambda=\left[\lambda_{1}, \lambda_{2}, \cdots, \lambda_{m-1}\right]$ needs to be optimized so that we can make

$$
\min E\left[\left|\hat{s}_{j}(t)\right|\right] \text { set up: } \quad \min E\left[\left|x_{0}(t)-\lambda y(t)\right|^{2}\right]
$$

Derivative (7), $\frac{\partial E\left[\left|x_{0}(t)-\lambda y(t)\right|^{2}\right]}{\partial \lambda}=0$, get $\lambda=E\left[x_{0}(t) y(t)^{T}\right]\left[E\left(y(t) y(t)^{T}\right]^{-1}\right.$.So the no,j source is:

$$
\begin{aligned}
& \hat{s}_{j}(t)=\left(a^{j}\right)^{T} x(t)-\left(a^{j}\right)^{T} E\left[x(t) y(t)^{T}\right]\left[E\left(y(t) y(t)^{T}\right)\right]^{-1} y(t) \\
& w_{j}=\left(a^{j}\right)^{T}-\left(a^{j}\right)^{T} E\left[x(t) y(t)^{T}\right]\left[E\left(y(t) y(t)^{T}\right)\right]^{-1} B
\end{aligned}
$$

From the formula (8) it is known that the source is only related to the observed signal and the corresponding basis vector, which can be directly calculated without iteration.

According to the paper ${ }^{[7]}$, the theorem can be proved:

In linear mixing, the basis vectors of the no.j source signals,if $\operatorname{rank}\left(A_{j}\right)=\mathrm{m}-1$,then $w_{j}=\left(a^{j}\right)^{T}-\left(a^{j}\right)^{T} E\left[x(t) y(t)^{T}\right]\left[E\left(y(t) y(t)^{T}\right)\right]^{-1 B}, \hat{s}_{j}(t)=w_{j} x(t)$ is completely consistent with the source signal $s_{j}(t)$.At this point you can get the source signal $s_{j}(t)$. The above method is called the two step blind extraction method under the condition of non complete sparse.

\section{Simulation experiment}

The simulation of this algorithm is carried out to prove the superiority of the algorithm compared to other anti collision algorithm.

\subsection{Experiment 1: feasibility experiment}

The ASK signal source signal1.wav is used as the source of the signal source, and the noise source mix1.wav is used as the source of noise.The length of both the signal source and the noise source are 10S.In the MATLAB2010 environment, the simulation is carried out in the frequency domain, the single source region sample is detected, and the base vector is estimated.Simulation results are shown in Figure 4.1. 


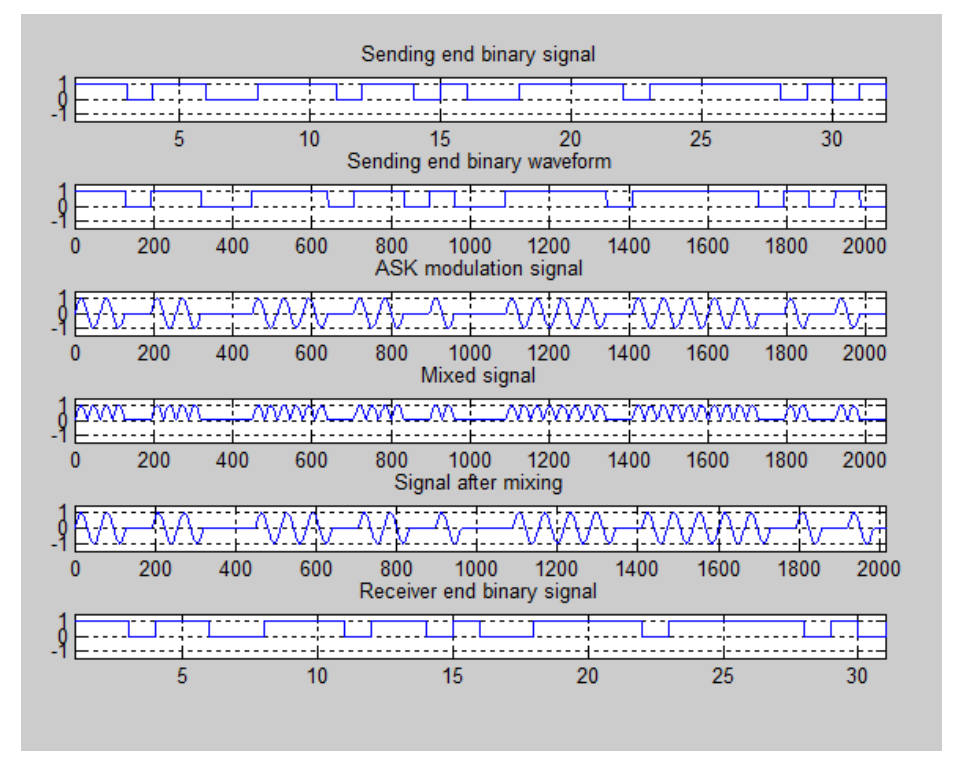

Fig4.1Signal separation feasibility experiment

From the experimental results, we can know that this paper can effectively separate the source signals based on the algorithm of the blind, to achieve the effect of the collision detection and the separation of the signal.

\subsection{Experiment 2: throughput analysis experiment}

The throughput calculation formula of ALOHA algorithm and time slot ALOHA algorithm are respectively:

$$
\begin{gathered}
S_{A L O H A}=G \cdot e^{-2 G} \\
S_{F S A}=G \cdot e^{-G}
\end{gathered}
$$

A blind separation method is used to label the anti collision is the use of a read and write to complete a number of tags read.The reader with this algorithm can complete the identification of the tag signal if it has multiple antennas and enough computing power.The number of tags to be reached within a time slot is $\mathrm{n}$, and the reader is installed with a $\mathrm{M}$ receiving antenna, and the number of tags that can be correctly identified is N.The throughput of the system in which the system can be successfully expanded in a certain time slot is:

$$
T=\sum_{n=0}^{\infty} n_{s} P_{n}
$$

$n_{s}$ is the average label number which can be successfully read by the reader:

$$
n_{s}=n\left(1-\frac{1}{N}\right)^{n-1}
$$

When the tag is enough in the reader area, it can be considered as a Poisson distribution, and the distribution of the label is:

$$
P_{n}=\left(G^{n} / n !\right) \exp (-G)
$$

Take (13),(14)into(12),we can get the throughput based on the blind source separation algorithm in this paper.

$$
T=G \exp \left(-\frac{G}{N}\right)
$$


Through the above analysis, we can compare the proposed algorithm with time slot ALOHA algorith $\mathrm{m}$ and the common blind source separation BSS algorithm, which is based on the MATLAB simulation, and compares the throughput.

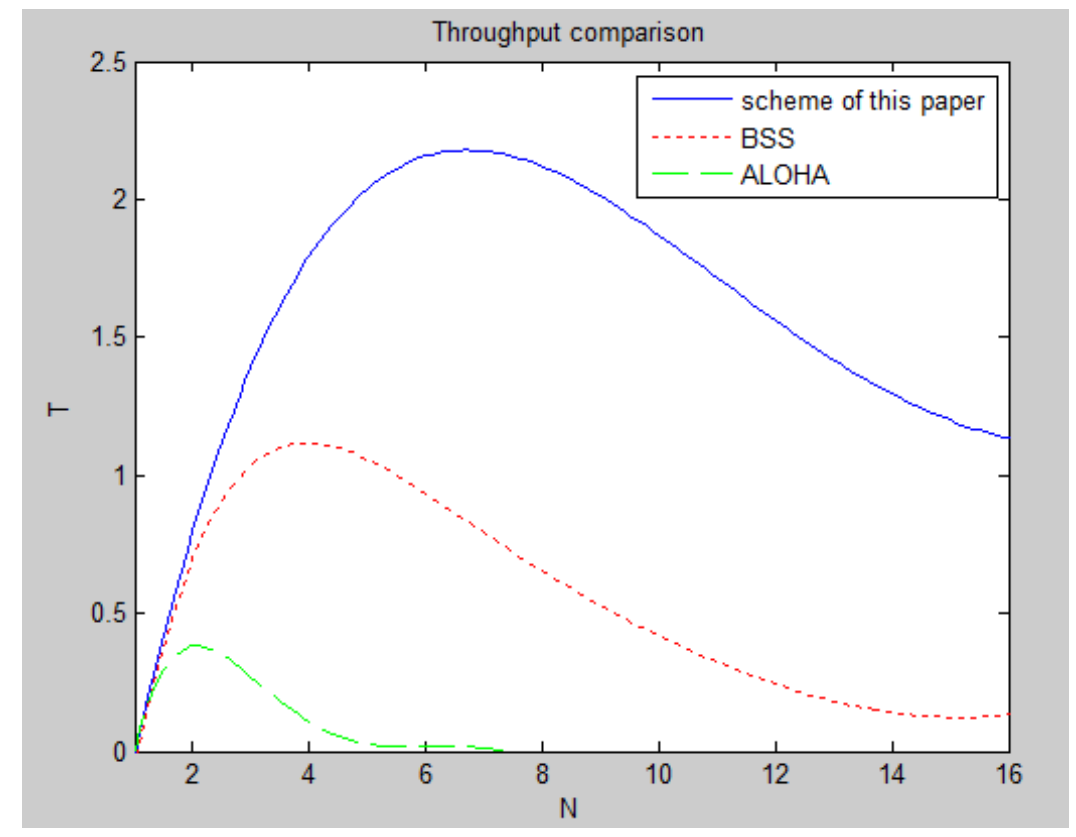

Fig4.2 Throughput comparison of three algorithms

From the above analysis, the throughput, the proposed algorithm is better than the BSS algorithm and ALOHA algorithm. When the reader antenna number is 3 , in the same time slot, the throughput of the algorithm as the tag arrival rate increases with increasing and achieve to reach the maximum value when the number of tags is 6.The throughput of the algorithm begins to decrease when the number of tags on the arrival is increased, which is because the number of tags currently being able to be separated is 6 when using 3 antennas.In the stable region, the throughput of this scheme is better than the traditional BSS algorithm and ALOHA algorithm, the hardware overhead is small, and it is more suitable for dealing with the problem of RFID.

\section{Conclusion}

In this paper, based on the RFID anti collision model,in the light of the characteristics of RFID signal that is not completely sparse signal,we used signal unit interval to improve the method of blind separation and proposed a sparse set conditions in anti collision algorithm for blind separation of RFID.Under the condition that the number of tags is more than the number of readers,through simulation, the throughput of the scheme was proved to be better than the blind separation algorithm and ALOHA algorithm, which can be used to separate the signal from the impact signal.

\section{Acknowledgments}

As the research of the thesis is sponsored by National Natural Science Foundation of China (No: 61262075) and major scientific research project of Guangxi higher education (No: 201201ZD012) and Project Foundation of Guangxi Experiment Center of Information Sciencep (No: 20130206), we would like to extend our sincere gratitude to them. 


\section{Reference:}

[1]FINKENZELLER K:RFID-Handbook Fundamentals and Application in Contactless Smart Cards and Identification 2nd Edition New York;Wiley and Sons,2003.

[2]W. Zein-Elabdeen,E. Shaaban:An Enhanced Binary tree Anti-collision Technique for Dynamically Added Tags in RFID Systems. International Conference on Computer Engineering and Technology, Chengdu,China,2012.Vol 7:349-353.

[3]Xuhai Wang:Research on RFID anti collision based on specific encoding mode[D],Nanjing University of Posts and Telecommunications(2013).

[4]Li Hua,Wang Hong-jun.ICA-based UHF RFID multitag hybird data blind separation,Washington D.C:SPIE,Vol.210-217(2012).

[5]Fengbo LU,Zhitao Huang:Underdetermined Blind Source Separation(BSS):A Time-Frequency Approach[J].Electronic journal,Vol.39(2011),No.9.

[6]Ming Xiao,Shenli Xie,Yuli Fu:A delay based on the blind source separation based on frequency domain single source.Chinese Electronic journal[J],Vol 35(2007),p.37-41.

[7]Shenli Xie,Gongxun Sun:Sparse and incomplete blind signal extraction.Chinese Electronic journal[J],Vol.38(2010),No.5. 